\title{
Treatment Of Cardiorenal Syndrome Type 1: Ultrafiltration vs. Boluresis
}

\author{
Cihan Cevik, MD
}

One third of patients with acute decompensated heart failure also develop worsening kidney function, and the management of these patients is very challenging. This common clinical condition is now termed "Cardiorenal Syndrome Type 1". In this issue of the Journal, Omar and Zedan reviewed the subtypes, prevalence, pathophysiology, treatment, and outcomes of this complex syndrome. ${ }^{1}$ The authors should be congratulated for their effort to compile the current data on these syndromesfor which the best management is still a mystery.

Among the five different subtypes of cardiorenal syndrome, cardiologists frequently help manage patients with Type 1 . These patients usually receive high doses of diuretics or less commonly are treated with venovenous ultrafiltration. Current heart failure guidelines recommend ultrafiltration in these patients as a Class $1 \mathrm{la}$ recommendation. ${ }^{2}$ However,limited data are available comparing the safety and efficacy of ultrafiltration with diuresis in acute heart failure complicated with cardiorenal syndrome and congestion. Recently, the CARRESS-HF trial demonstrated that the best treatment for these patients was not ultrafiltration. ${ }^{3}$ This randomized trial included 188 patients with acute decompensated heart failure and worsening kidney function $(0.3 \mathrm{mg} / \mathrm{dl}$ increase in creatinine levels). The mean age of these patients was 68 years, $75 \%$ of the patients were men, and the average creatinine was $2 \mathrm{mg} / \mathrm{dl}$. Patients received either ultrafiltration therapy or aggressive diuresis with a goal of 3-5 liters of urine output per day. Patients randomized to ultrafiltration had higher rates of adverse events, mainly kidney failure, bleeding complications, and intravenous catheter related complications, than patients randomized to the medical treatment arm ( $72 \%$ vs. $57 \%, p=0.03)$. Aggressive diuretic treatment was superior to ultrafiltration in bivariate primary endpoints, including change in weight and change in serum creatinine levels at 96 hours after enrollment. Furthermore, ultrafiltration worsened the kidney func- tion more frequently than medical therapy in this trial. This investigator driven, National Heart, Lung, and Blood Institute funded trial is published online in the New England Journal of Medicine in November 2012.

The diuretic doses used in the pharmacologic arm of the CARRESS-HF trial need to be highlighted. The investigators suggested the use of significantly higher doses of intravenous loop diuretics with or without metolazone than is usual in clinical practice. For instance, the suggested dose for a patient who was on less than $80 \mathrm{mg}$ of oral loop diuretic daily before the cardiorenal syndrome was $160 \mathrm{mg}$ intravenous loop diuretic per day. If the urine output for this particular patient was less than three liters at 24 hours, the suggested dose was $320 \mathrm{mg}$ intravenous loop diuretic and $5 \mathrm{mg}$ metolazone daily. If the urine output was still less than three liters at the next 24 hours, the subsequent step was $560 \mathrm{mg}$ intravenous loop diuretic plus $10 \mathrm{mg}$ of metolazone and the consideration of dopamine, dobutamine, nitroglycerine, or nesiritide depending on the systolic blood pressure, symptoms, and ejection fraction. This strategy reminded me of the term "boluresis" which was initially thought to be harmful by increasing the creatinine and further worsening the kidney function. I suggest all clinicians treating these patients should be aware of the details on doses in the stepped pharmacologic care arm of CARESS-HF trial (found in the Supplementary Appendix of the original publication).

There is urgent need to find the best strategy to manage acute cardiorenal syndrome. From the CARESS-HF trial we learn that ultrafiltration should not be recommended as first-line, routine treatment for patients with Type 1 cardiorenal syndrome. I am anticipating that the next heart failure guidelines will consider the results of this study and downgrade the recommendations for ultrafiltration in acute decompensated heart failure patients. 


\section{REFERENCES}

1. Omar S, Zedan A. Cardiorenal Syndrome.The Southwest Respiratory and Critical Care Chronicles 2012; 1(1). 2. Hunt SA, Abraham WT, Chin MH, et al. 2009 Focused update incorporated into the ACC/AHA 2005 Guidelines for the Diagnosis and Management of Heart Failure in Adults: a report of the American College of Cardiology Foundation/ American Heart Association Task Force on Practice Guidelines: developed in collaboration with the International Societyfor Heart and Lung Transplantation. Circulation 2009; 119:e391-e479. [Erratum, Circulation 2010; 121(12) e258.] 3. Bart BA, Goldsmith SR, Lee KL, Givertz MM, O'Connor CM, Bull DA, Redfield MM, Deswal A, Rouleau JL, LeWinter MM, Ofili EO, Stevenson LW, Semigran MJ, Felker GM, Chen $\mathrm{HH}$, Hernandez AF, Anstrom KJ, McNulty SE, Velazquez EJ, Ibarra JC, Mascette AM, Braunwald E; Heart Failure Clinical Research Network. Ultrafiltration in decompensated heart failure with cardiorenal syndrome. $N$ Engl J Med. 2012; 367:2296-304.

\footnotetext{
Author Affliciation : Texas Heart Institute at St. Luke's Episcopal Hospital, Department of Adult Cardiology, Baylor College of Medicine, Houston, TX 77030

Author Contact Information: cevik@bcm.tmc.edu DOI: 10.12746/swrccc2013.0101.002
} 